\title{
EFFECTIVE APPROACh FOR DATA AgGregation in Wireless Sensor NeTwork: A STRUCTURe Free APPROACH
}

\author{
Umashankar Prasad $^{1}$, Dr. D.S. Adane ${ }^{2}$ \\ ${ }^{1}$ Department of Computer Science and Engineering \\ Ramdeobaba College of Engineering and Management \\ Nagpur (M.S.) India \\ prasadud@rknec.edu \\ ${ }^{2}$ Department of Information Technology \\ Ramdeobaba College of Engineering and Management \\ Nagpur (M.S.) India \\ hodit@rknec.edu
}

\begin{abstract}
Wireless Sensor Network consist of thousands sensor node which have limited Power, Computation, Sensing and Communication capabilities. Among all operation of Sensor Node, Wireless Communication consumes most of the energy. So it is necessary to decrease the number of packets transmitted through the network. Many Sensor Node could detect similar event, which increases the overall bandwidth utilization to transmit redundant data. Here Nodes computation is cheaper than communication in terms of energy. So the technique of Data Aggregation is applied to summarize data which decreases the amount of data transmitted in the network, which in turn increases the lifetime of the network. Many Data aggregation protocols are based on a structured approach which is suitable for data collection application. But maintenance of the structure is an extra overhead and this approach is not suitable for dynamic scenario. So we propose an ad-hoc data aggregation protocol for dynamic scenario mainly event based application.
\end{abstract}

Key words: Wireless Sensor Networks, throughput, delay, energy efficiency, protocols, event based, dynamic, Data Aggregation.

\section{Introduction}

Wireless Sensor Networks are composed of many inexpensive, lowpowered sensing devices with limited computational, memory and communication resources [1], [2]. This networks offer various low-cost solution to 
various problem including target tracking, environment and health monitoring, traffic regulation and wildfire detection. Since sensor node are low cost it had simple hardware with many resource constraint. Hence it is challenging to provide to gather data among these battery powered nodes, since node battery is limited. To reduce power consumption various methods like radio scheduling, topology control, control packet elimination and the most important data aggregation is used [2], [3]. Data Aggregation protocol aims at summarize data packets of different nodes so the total amount of data transmitted over the network could be minimized and bandwidth utilization also increases which in turn affects the through-put of the network.

Optimal Data Aggregation can be measured in terms of energy consumed by transferring the collected information to sink. But it heavily depends on the topology of network, placement of data aggregator nodes, event source, etc. Many centralized structured approach [4], [5], [6], [7], [8], [9] are mainly focused on data gathering application where all nodes report their sensor value periodical to Sink. Many distributed structured approach [10], [11], [12] are proposed for event based application. There are various limitation for using structured approach in event based application where event region change is frequent, First overhead of construction and maintenance of structure is not practical is dynamic scenario. Second absents of center of event source leads to in-optimal structure formation for aggregation. Third centralized computation of structure is not possible in dynamic scenario.

In this paper we focus on structure free data-aggregation mainly used for event based application. The purpose is to redesign DAA [13] (i.e. Data Aware Anycast) structure free data aggregation protocol to improve the lifetime of the network. There are two major challenges in structure free data aggregation. First, who should send data to whom for aggregation? Second, who should wait for whom before sending so optimal aggregation could be achieved? Fan [13] has divided the problem in two part spatial convergence and temporal convergence and had developed two approach to tackle it. DAA protocol tackles spatial convergence problem thereby solving the first routing challenge. RW (i.e. Randomized Waiting) protocol at application layer tackles temporal convergence solving second who should for whom challenge.

We propose a new model for Structure free data aggregation which combines the Mac-Layer protocol Data Aware Anycast (DAA) and Sensor Mac (S-MAC) and Application Layer protocol Randomized Waiting (RW). Based on observation combining the basic S-MAC protocol with DAA protocol improves the lifetime as compared with DAA [13] original protocol. 


\section{Related Work}

In wireless sensor network, Nodes communication cost is far more expensive then the computation cost. Pottie and Kaiser [14] research said that energy involved in computing lots of instruction is equivalent to transmitting one packet over wireless channel, therefore data aggregation while routing the packets is very important to extend the lifetime of the network. Various protocol are proposed for in-network data aggregation purpose which are divided in broad category as Structure-Based and Structure Free.

\subsection{Structured-Based Approach}

In structure based approach there is construction of a network structure which enable the nodes to know which node should wait for whom before sending its own data so that node can aggregate data received from others. So there is an overhead of construction and maintenance of a global network structure which could be done centralized or distributed manner.

In cluster based network all sensor nodes are not homogeneous in terms of responsibility. Few nodes becomes cluster head and rest all remain sensor node. Every node belong to particular cluster and Each nodes send data to their sensor data to their respective cluster head who aggregates all the received data and send it to base station. Heinzelman introduced LEACH [15] protocol which has two stages of operation. First stage is setup phase in which whole network is organized is cluster and cluster head is selected and second stage is steady phase in which all nodes send data to cluster heads then cluster head aggregates data from all nodes and send aggregated packet to sink directly by high power transmitter. Cluster head is re-elected after some time interval since the high power transmission to sink consumes more. One may say that it should save more energy by aggregating then transmission to sink.

Lindsey introduced protocol PEGASIS [16] where the nodes organized themselves in chain pattern. The farthest node initiate the chain formation and then rest of neighboring nodes form a linear structure. While data gathering each node send data to neighbor node which aggregates it and send to next in linear fashion. Both LEACH and PEGASIS assumes that the sink is could be reached in one hop which poses some physical limitation on the network.

Tree-Based data routing protocol [17], [18] sensing information entropy for data compression (aggregation). They assume global knowledge of each nodes entropy information. 


\subsection{Structure-Free Approach}

In this approach there is no need to maintain a global structure for data aggregation. Data aggregation done is such network may not be optimal when compared to structure approaches but the overhead of construction and maintenance is absent which is good for event based application where the event region changes frequently.

Major work done in this category is by Kai-Wei Fan. In this paper [13] author had observed the opportunity for data aggregation and had proposed DAA mac layer protocol and RW application layer protocol is do so. Which makes routing and aggregation decision dynamically.

Chao [19] have proposed SFEB protocol which extends the DAA approach and is suitable for specific network scenarios made improvements to it.

\section{Spatial and Temporal Convergences}

For optimal aggregation, all nodes should transmit in certain order. All structured data aggregation approach are designed to follow ordering of packets while forward it. Structured approach are well suited for data gathering application where every node has data to report and they form an hierarchy where leaves node send their data intermediate nodes wait and receives from all child packet then aggregates then forward it. These approaches have high overhead of maintenance of structure and are not suitable for event based application. For data aggregation in event based application is done only when the packets happen to meet at same node at same time with potential of aggregation. We study and design structure free approach for data aggregation well suited for event based application to avoid the overhead of maintenance of structure.

Spatial and temporal convergence are necessary condition for data aggregation. For structure free approach we would like to extend the work done by Kai-Wei Fan [13]. We would like to combine benefit S-MAC and DAA for greater efficiency and improved lifetime.

Main advantage of structure free approach are, First it is tolerance to dynamic events where the event region changes frequently. Second it is fault tolerance, nodes will aggregate and forward data till there is at least one node in its range. Third it is robust to mobility of nodes till it's in range of other nodes. Forth it is robust to interference, if there is failure while sending data it can retry in next cycle as long as node has power. 


\subsection{Spatial Convergence}

In this section we are presenting the new scheme which combine S-MAC and DAA. For proper data aggregation global knowledge of few things are needed but that should be network topology. The protocol defined here need to have following assumption:

- Nodes need to know their own location and sink location. This could be done by localization protocol [20], [21] or GPS devices.

- Nodes are need to be time synchronized for coordinative sleeping required by S-MAC and for aggregating packets generated at same time or different parameter could be considered for aggregation of data.

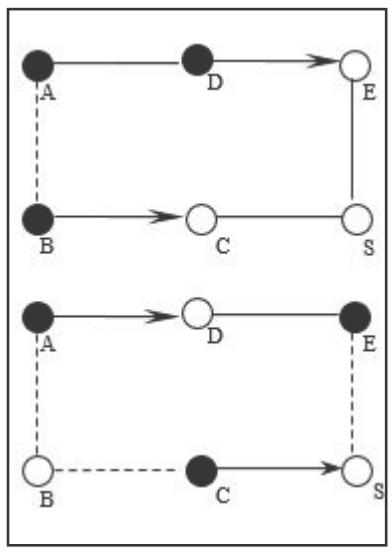

(a)

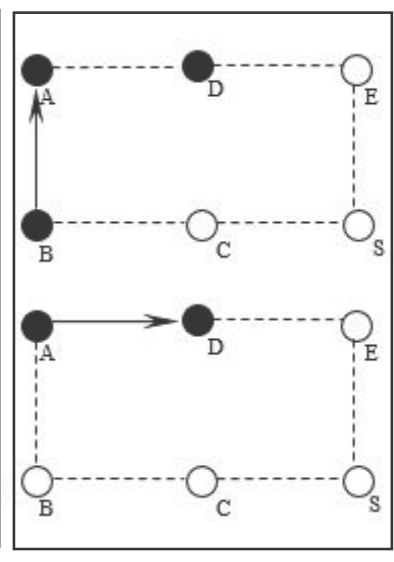

(b)

Figure 1. Showing an opportunity for aggregation. (a) Nodes without data aggregation send data along the route without checking the opportunity for aggregation. (b) Node got to know that one hop neighbor has opportunity for aggregation so send data along the path where aggregation is possible.

Figure 1a shows the scenario shows how data will be forward if the opportunity in aggregation is ignore and data is transmitted normally. Figure $1 \mathrm{~b}$ show if node B knows that its hop neighbor node A has data that could be aggregated in future so node B forward data to node A instead of node $\mathrm{C}$ which happened in general condition. 


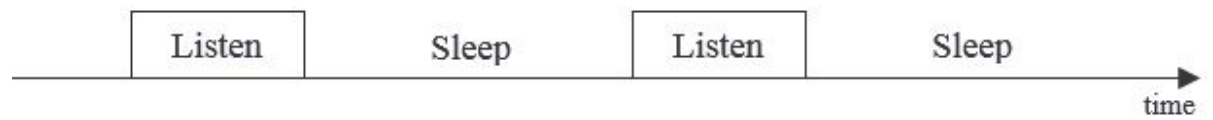

Figure 2. Each duty cycle is divide in listening period and sleep period and listening period is synchronized with all node taking part in network.

First we are using S-MAC as lower mac layer in our protocol. S-MAC has an adaptive sleeping schedule which need synchronization which is one of our assumption. S-MAC has a duty cycle (DC) which is sub-divided into two parts listening period and sleeping period as show in figure 2. Duty cycle of all nodes are synchronized such that one can send data to its neighboring on its listening period only.

Data exchange takes place in a manner as RTS/CTS/DATA/ACK. For sending data across nodes RTS and CTS exchange must be happen in listening period so both sender and receiver know the data is coming then DATA/ACK could be exchanged over the sleep period and all the rest nodes not taking part in transmission can go to sleep as the listening period end. Here Duty cycle must be selected such that data transmission should be completed before the next listening slot begins. Carrier sense before sending any packet and the handshake model avoid the hidden and exposed terminal problem of wireless channel. Network Allocation Vector (NAV) could be send along the pack to alert other sensing nodes to back off certain amount of time.

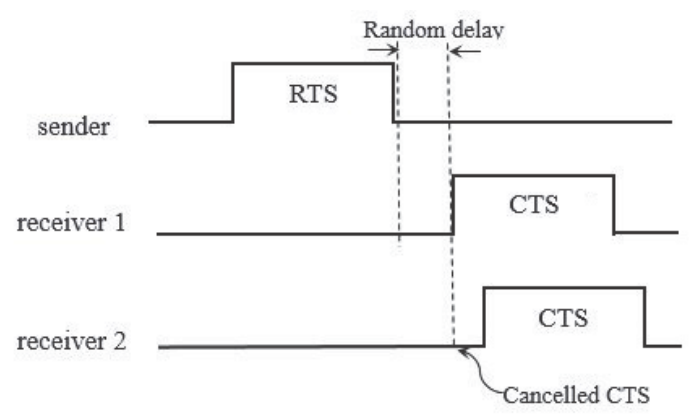

Figure 3. Showing how multiple CTS is cancelled

Second section of protocol is DAA based at MAC layer which decide the next hop for each transmission. It was RTS packet is decide which node is ready to receive transmission. RTS is send during the listening phase of SMAC. The opportunity for aggregation in the next hop could be decided by Aggregation ID (AID). AID could be the timestamp of event so same event detected by multiple node could be aggregated or could be anything depending upon the applications. RTS packet contains AID within it and nodes re- 
ceiving such RTS packet also has data with same AID so there is opportunity for aggregation. This way only those nodes with got AID match will reply with CTS. But the same RTS packet is received by more than one node and both the node reply to one RTS then there will be CTS collision to solve that. We delay the CTS with some minor random time to avoid that and channel is sensed first before sending CTS. So in this way subsequent is cancelled as shown in Figure 3. To increase aggregation. We will reply to RTS with CTS the receiving node is closer to sink then sender node.

Nodes are categorized into three classes and given priority. So higher priority nodes reply with CTS early then lower priority. These three classes of priority are as follows:

- Class A: Receiving node has same AID as in RTS and closer to sink then sender.

- Class B: Receiving node has same AID as in RTS and farther to sink then sender.

- Class C: Receiving node does not have has same AID as in RTS but it's closer to sink then sender.

Class A has more priority then Class B. Class B has more priority then Class C. So if a node receives a RTS it checks that to which class it belong and then reply CTS is delayed accordingly. So the class A send CTS first then other two class.

The information that each node have is the distance to sink which could be used as the routing parameter. This parameter in incorporated least priority in reply CTS for proper routing to sink.

\subsection{Temporal Convergence}

Aggregation only happens when two packet with same AID meet at same node and at same time. So if we transmit the data as soon as it is captured by sensor then some packets will be forward immediately and the chances of aggregation will be lost. Same AID of data generally exist among the data captured for same physical event by many nodes. If all nodes send data immediately by the above method there will be only few aggregation. So nodes need to wait for time before sending their own data so chances of aggregation is maximized. Since there is no structure this could be done in random manner. So random waiting (RW) at application layer is solution to it. Here nodes has a random wait period at the start of listening period before sending RTS to avoid collision as well as to solve temporal Convergence issue. However this method does not guarantee optimal aggregation due to its random nature but increases aggregation chances. This enables few nodes to wait few duty cycle before sending when they receive RTS from neighbor node before their own RTS gets ready. 
The random value range selection plays crucial role in optimal aggregation that depends on the density of network, event size, time to transmit, listening and sleeping period chosen in S-MAC.

\section{Simulation Scenario}

To justify my design of the above protocol we need to compare it with non-aggregated and structured based approaches of data aggregation at least in simulation environment. We have chosen Ptolemy II simulator for justifying our design. Ptolemy II has all basic component for wireless network simulation and each and every component is customized from its wide actor set. It is an open-source software framework supporting experimentation with actororiented design. The model semantics in Ptolemy II is not dependent on framework but by a software component called director. It's developed and supported by UC Berkeley EECS dept. [22].

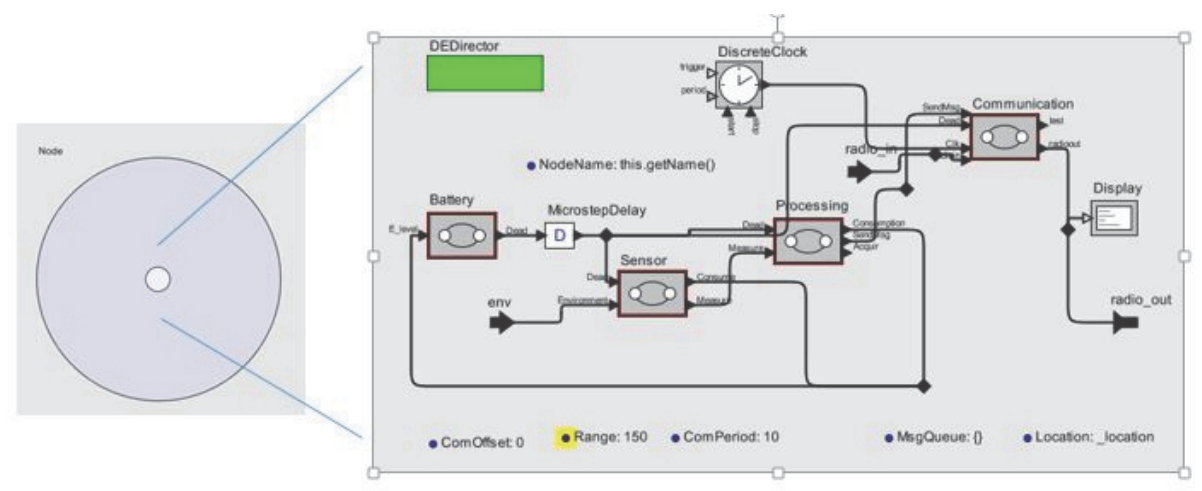

Figure 4. Sensor Node design in Ptolemy II. 


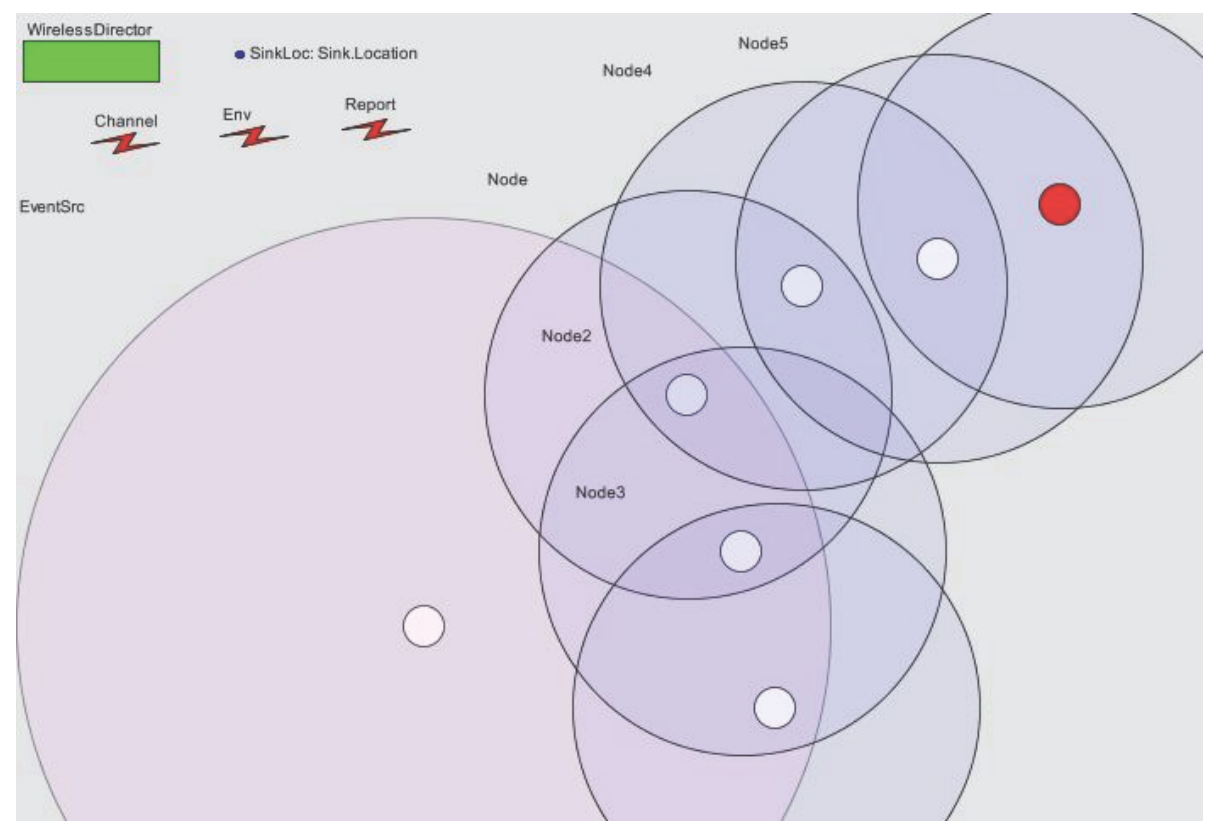

Figure 5. Simulation scenario for one event.

Each sensor node in our simulation is actor in wireless director environment. Sensor node is broken in four component as shown in figure 4. All four component design is based on finite state machine (FSM) where each state has refinements wherever needed.

A typical scenario is shown in figure 5 in which biggest circle on left is event source with its range. A sensor node is show with white circle with larger circle around it to represent the range that node covers. The node with red Center is sink node where the information need to be sent. Three nodes that fall in event region senses that event and try to report and broadcast RTS and the neighbor nodes in event region have higher priority as the AID could match reply with CTS early then not matching AID nodes outside the event region. So these three node aggregate the data among each other in three cycle and then multi-hop that one packet to red sink.

\section{Future Work}

The performance analysis of the above model need to done against the non-aggregated and structured based data aggregation approach. The report generation and analysis method are need to be incorporated in the proposed approach. 


\section{Conclusion}

The lifetime of the network is maximized by introducing the S-MAC layer functionality over the DAA protocol. S-MAC basically solves the issue of idle listening of the wireless channel. So nodes has only to listen to channel in the listening cycle. This improvement over tradition DAA approach saves a lot more energy thereby increasing the lifetime. But it also increase per-hop delay, so there should be tradeoff between the delay and energy saved in this structure free aggregation method.

\section{Acknowledgments}

Editors of the JACSM would like to thank authors and reviewers for their interest in the subject of JACSM, contributions to the journal and their great job which enables to create contents of the journal's volumes.

\section{References}

1. Akyildiz, I. F., Su, W., Sankarasubramaniam, Y., \& Cayirci, E. (2002). Wireless sensor networks: a survey. Computer networks, 38(4), 393-422.

2. Yick, Jennifer, Biswanath Mukherjee, and Dipak Ghosal. "Wireless sensor network survey." Computer networks 52.12 (2008): 2292-2330.

3. Akkaya, Kemal, Murat Demirbas, and R. Savas Aygun. "The impact of data aggregation on the performance of wireless sensor networks." Wireless Communications and Mobile Computing 8.2 (2008): 171-193.

4. Kulik, Joanna, Wendi Heinzelman, and Hari Balakrishnan. "Negotiation-based protocols for disseminating information in wireless sensor networks." Wireless networks 8.2/3 (2002): 169-185.

5. Intanagonwiwat, Chalermek, Ramesh Govindan, and Deborah Estrin. "Directed diffusion: a scalable and robust communication paradigm for sensor networks." Proceedings of the 6th annual international conference on Mobile computing and networking. ACM, 2000.

6. Krishnamachari, Bhaskar, and John Heidemann. "Application-specific modelling of information routing in wireless sensor networks." Performance, Computing, and Communications, 2004 IEEE International Conference on. IEEE, 2004.

7. Heinzelman, Wendi Beth. Application-specific protocol architectures for wireless networks. Diss. Massachusetts Institute of Technology, 2000.

8. Lindsey, Stephanie, Cauligi Raghavendra, and Krishna M. Sivalingam. "Data gathering algorithms in sensor networks using energy metrics." Parallel and Distributed Systems, IEEE Transactions on 13.9 (2002): 924-935. 
9. Ding, Min, Xiuzhen Cheng, and Guoliang Xue. "Aggregation tree construction in sensor networks." Vehicular Technology Conference, 2003. VTC 2003-Fall. 2003 IEEE 58th. Vol. 4. IEEE, 2003.

10. Zhang, Wensheng, and Guohong Cao. "DCTC: dynamic convoy tree-based collaboration for target tracking in sensor networks." Wireless Communications, IEEE Transactions on 3.5 (2004): 1689-1701.

11. Zhang, Wensheng, and Guohong Cao. "Optimizing tree reconfiguration for mobile target tracking in sensor networks." INFOCOM 2004. Twenty-third AnnualJoint Conference of the IEEE Computer and Communications Societies. Vol. 4. IEEE, 2004.

12. Intanagonwiwat, C., Estrin, D., Govindan, R., \& Heidemann, J. (2002). Impact of network density on data aggregation in wireless sensor networks. In Distributed Computing Systems, 2002. Proceedings. 22nd International Conference on (pp. 457-458). IEEE.

13. Fan, Kai-Wei, Sha Liu, and Prasun Sinha. "Structure-free data aggregation in sensor networks." Mobile Computing, IEEE Transactions on 6.8 (2007): 929942.

14. Pottie, Gregory J., and William J. Kaiser. "Wireless integrated network sensors." Communications of the ACM 43.5 (2000): 51-58.

15. Heinzelman, Wendi Rabiner, Anantha Chandrakasan, and Hari Balakrishnan. "Energy-efficient communication protocol for wireless microsensor networks." System Sciences, 2000. Proceedings of the 33rd Annual Hawaii International Conference on. IEEE, 2000.

16. Lindsey, Stephanie, and Cauligi S. Raghavendra. "PEGASIS: Power-efficient gathering in sensor information systems." Aerospace conference proceedings, 2002. IEEE. Vol. 3. IEEE, 2002.

17. Scaglione, Anna, and Sergio Servetto. "On the interdependence of routing and data compression in multi-hop sensor networks." Wireless Networks 11.1-2 (2005): 149-160.

18. Scaglione, Anna. "Routing and data compression in sensor networks: stochastic models for sensor data that guarantee scalability." Information Theory, 2003. Proceedings. IEEE International Symposium on. IEEE, 2003.

19. Chao, Chih-Min, and Tzu-Ying Hsiao. "Design of structure-free and energybalanced data aggregation in wireless sensor networks." High Performance Computing and Communications, 2009. HPCC'09. 11th IEEE International Conference on. IEEE, 2009.

20. Rudafshani, Masoomeh, and Suprakash Datta. "Localization in wireless sensor networks." Information Processing in Sensor Networks, 2007. IPSN 2007. 6th International Symposium on. IEEE, 2007.

21. Chraibi, Youssef. "Localization in wireless sensor networks." (2005).

22. The Ptolemy Project. http://ptolemy.eecs.berkeley.edu/. 